\title{
The Role of Narcissism on Concerns for SNS Privacy: Vulnerable Narcissism in Threatening Situations
}

Elizabeth A. Kwolek

West Virginia University

Follow this and additional works at: https://researchrepository.wvu.edu/etd

\section{Recommended Citation}

Kwolek, Elizabeth A., "The Role of Narcissism on Concerns for SNS Privacy: Vulnerable Narcissism in Threatening Situations" (2012). Graduate Theses, Dissertations, and Problem Reports. 4884.

https://researchrepository.wvu.edu/etd/4884

This Thesis is protected by copyright and/or related rights. It has been brought to you by the The Research Repository @ WVU with permission from the rights-holder(s). You are free to use this Thesis in any way that is permitted by the copyright and related rights legislation that applies to your use. For other uses you must obtain permission from the rights-holder(s) directly, unless additional rights are indicated by a Creative Commons license in the record and/ or on the work itself. This Thesis has been accepted for inclusion in WVU Graduate Theses, Dissertations, and Problem Reports collection by an authorized administrator of The Research Repository @ WVU. For more information, please contact researchrepository@mail.wvu.edu. 


\title{
The Role of Narcissism on Concerns for SNS Privacy: Vulnerable Narcissism in Threatening Situations
}

\author{
Elizabeth A. Kwolek
}

\author{
Thesis submitted to the \\ Perley Isaac Reed School of Journalism \\ at West Virginia University \\ in partial fulfillment of the requirements \\ for the degree of
Master of Science
in
Journalism
Hongmin Ahn, Ph.D., Chair
Nicholas Bowman, Ph.D.
Elizabeth Oppe, Ph.D.
Steve Urbanski, Ph.D. \\ Morgantown, West Virginia \\ 2012
}

Keywords: Social Network Sites, Narcissism, Privacy, Threat, Regression Analysis

Copyright 2012 Elizabeth A. Kwolek 


\section{ABSTRACT \\ The Role of Narcissism on Concerns for SNS Privacy: Vulnerable Narcissism in Threatening Situations}

\section{Elizabeth A. Kwolek}

This study aims to identify psychological factors that predict one's likelihood to disengage with privacy-threatening activities on Social Networking Sites (SNS). Particularly, it pays special attention to the concept of narcissism and its impact on one's response to SNS privacy-threatening situations. By evaluating narcissism in two different ways — grandiose and vulnerable - the study attempts to provide a better understanding of the role of narcissism in SNS usage and concerns over privacy issues. Study I shows that vulnerable narcissism has a significant influence on consumers' likelihood of disengaging with privacy-threatening activities on SNS while grandiose narcissism has no influence. Self-esteem, computer anxiety, and concern for information privacy also have significant influence on consumers' responses to privacy-threatening events on SNS. Study II further suggests that when people experience interpersonal rejection threats on SNS, the effect of vulnerable narcissism on one's intention to disengage with privacy-threatening activity is largely attenuated. The findings from two studies offer insight into the psychological process by which narcissism influences privacy concerns over SNS in both macro and micro levels. 


\section{ACKNOWLEDGMENTS}

I wish to thank my chair, Dr. Hongmin Ahn for her constant challenges, support and encouragement. You have gone above and beyond the role of a committee chair, and have become my mentor during the time we have worked together. Thank you for extending your passion for research to me. Your dedication and patience have been instrumental to educating and guiding me through this process.

To the members of my committee: Dr. Nicholas Bowman, Dr. Elizabeth Oppe, and Dr. Steve Urbanski, thank you for your feedback during this process. I have been fortunate to have a flexible and well-rounded committee able to share their knowledge to assist with this research. Thank you for your time, flexibility, and support. 


\section{TABLE OF CONTENTS}

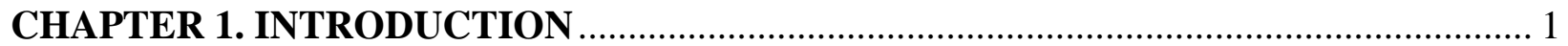

CHAPTER 2. THEORETICAL BACKGROUND ....................................................... 3

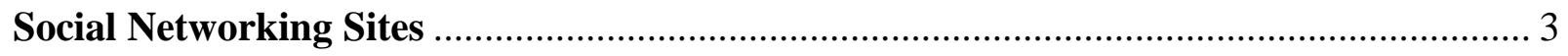

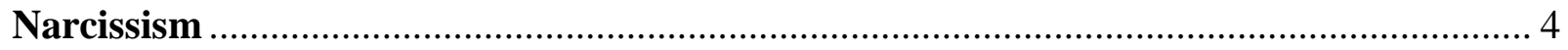

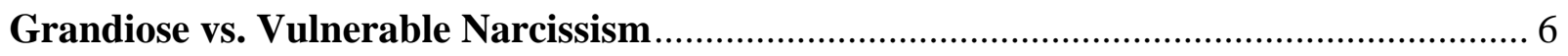

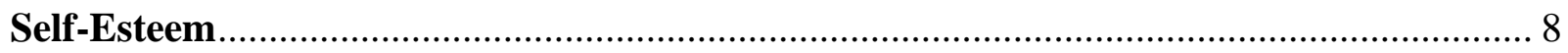

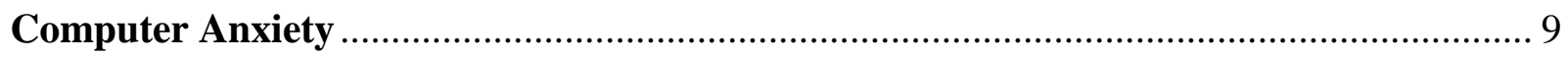

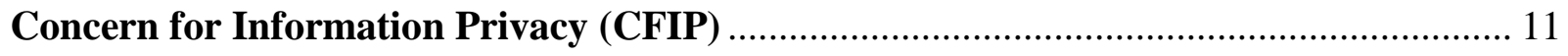

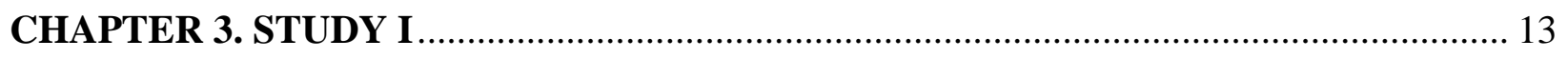

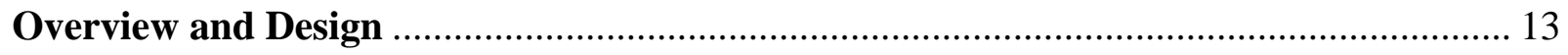

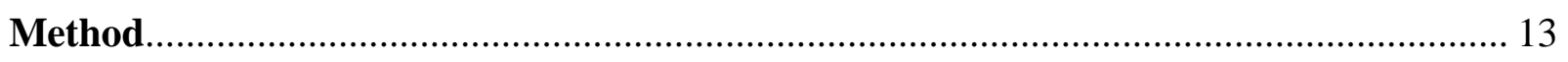

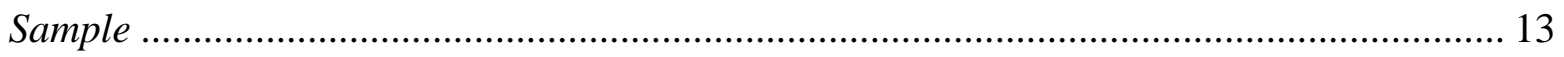

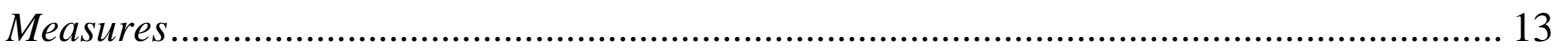

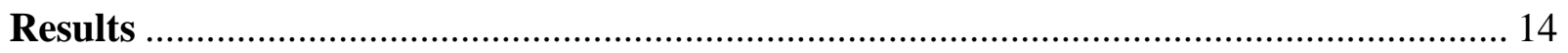

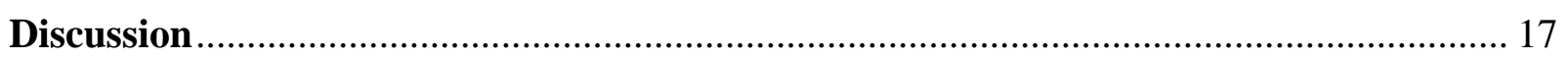

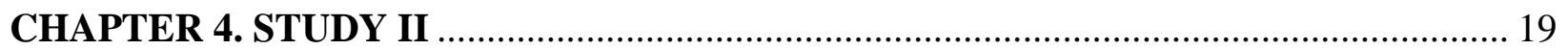

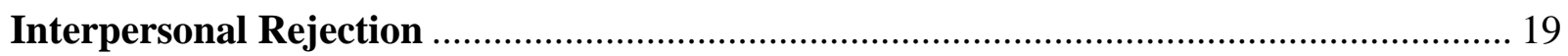

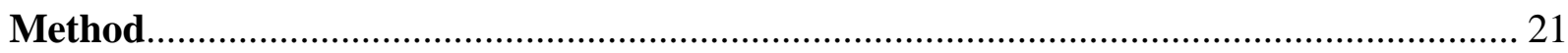

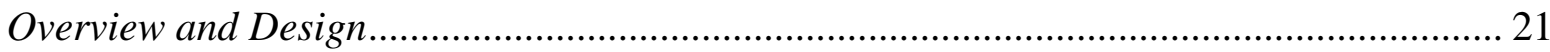

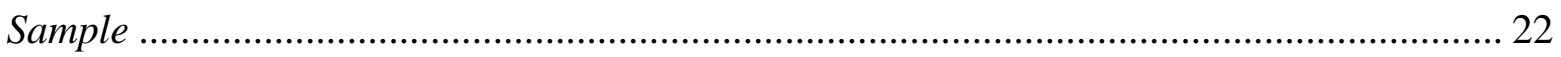

Stimuli Development and Pre-test .......................................................................... 22

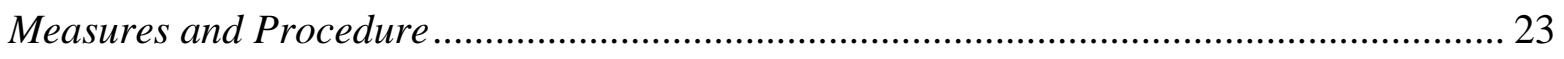

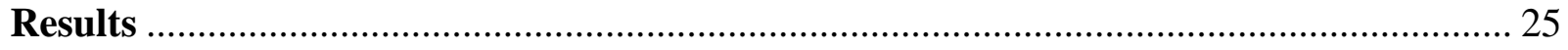

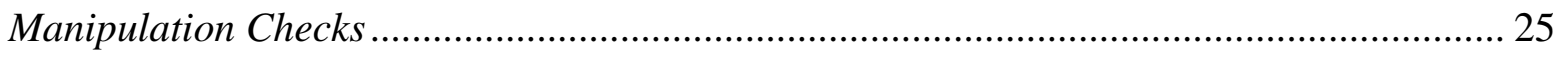

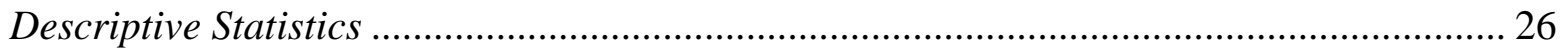

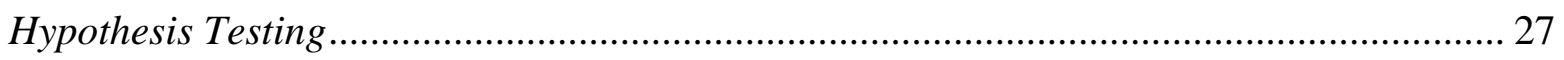

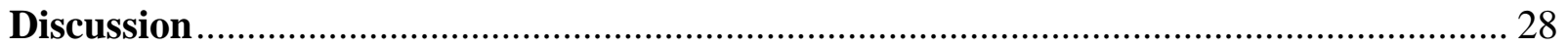

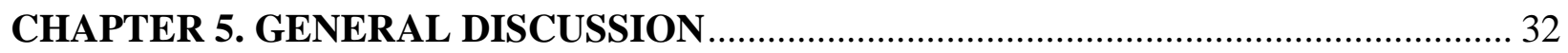

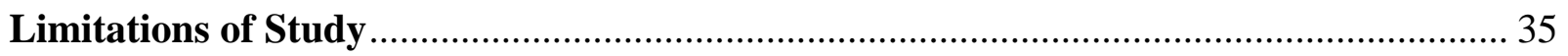

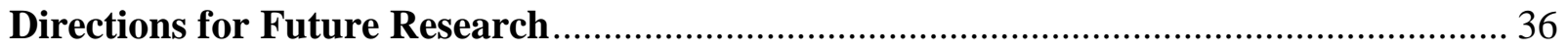

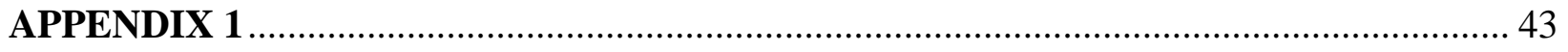

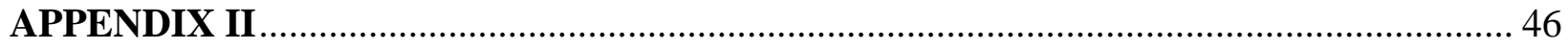




\section{CHAPTER 1. INTRODUCTION}

During the past few years, social networking sites (SNS) have become a catalyst for interpersonal communication on the Internet. Technological advances have allowed people to spread information at a very rapid rate and now, nearly everything a user does online is able to be broadcast in real-time for the entire world to view (Hof 2011). The immediacy and speed have helped SNS continue to expand in popularity (Kantar Media Company 2011). However, the ability to access and broadcast personal information on SNS introduces privacy concerns as well as security problems among SNS users. Given the large user base and the large amount of identity-relevant information on SNS, concerns about privacy have grown, and such concerns peaked in December 2009 when the popular SNS Facebook made all users' information publicly available (Rothery 2010). While the privacy and security issue in SNS has become an important topic in today's society, relatively little research has considered factors influencing individuals' responses to privacy-threatening activity on SNS usage.

To fill the void in SNS literature, this study aims to identify predictable psychological factors that will explain consumers' likelihood to disengage with privacy-threatening activities on SNS usage. Particularly, the study pays special attention to the concept of narcissism. While the impact of narcissism has been widely studied on SNS user behaviors (Bergman et al. 2011; Carpenter 2012; DeWall et al. 2011; Mehdizadeh 2010), the scope of narcissism, as a personality trait, has only been analyzed as one generalized construct. By evaluating narcissism in two different constructs - grandiose and vulnerable - this study attempts to provide a better understanding the role of narcissism has on SNS usage. For this purpose, Study I was developed to test the effects of grandiose and vulnerable narcissism on individual responses to privacythreatening activities on SNS. Study II was developed to confirm the link between vulnerable 
narcissism and response to privacy-threatening activity on SNS as well as to identify the impact of interpersonal rejection on the likelihood to disengage with SNS when introduced as a threatening SNS scenario. Findings from the two studies provide both theoretical and managerial implications regarding personality factors and their predictability to explain individual responses to SNS practices of attacking privacy. 


\section{CHAPTER 2. THEORETICAL BACKGROUND}

\section{Social Networking Sites}

SNS have expanded and grown in popularity over the past decade. In 2011, the top three SNS included the following: Facebook with approximately 156,347,820 unique visitors, Twitter with 34,748,861, and Linkedin.com with 23,828,271 (Kantar Media Company 2011). In addition to these statistics, data collected from a recent study revealed that of more than 300 surveyed subjects, each participant admitted to using SNS on a regular basis and to spending an average of approximately one hour a week online (Shin 2010).

Many SNS typically get initiated by a small group of people then, through word-ofmouth communication, membership grows as more and more people send invitations to join the networking site (Trusov, Bucklin and Pauwels 2009). What is unique about this new form of the Internet is that SNS are built upon universally shared experiences and include the perception of shared interests or missions. This communication strategy is appealing because it combines the possibility, "of overcoming consumer resistance with significantly lower costs and fast delivery — especially through technology, such as the internet" (Trusov, Bucklin and Pauwels 2009, p. 90).

While technological advances have allowed more information to spread quicker than ever, there are downfalls of them. For example, the concern for privacy is a growing issue as the SNS expands in popularity (Chen and Rea 2004). One anecdotal example was in 2006 when Facebook launched a site modification that created a threat to consumer privacy. Users formed groups to protest the new feature enabling user activity to be publicly viewed by other Facebook users through a development coined the "News Feed" (Boyd 2008). The feature made previously accessible information more easily exposed which caused users to lose their personal sense of 
privacy control (Boyd 2008). In less than a day, a response was made through a blog by Facebook's founder, Mark Zuckerberg, which informed users to rest assured that modifications would be made (Boyd 2008). While it was not the first time privacy became a concern for users and SNS corporate entities, it was one of the largest landmark events that spurred concern from all parties invested in SNS.

Given that privacy has grown into as a critical concern, there is a need for SNS studies to address potential psychological mechanisms that explain how SNS users respond to the collection and usage of personal information. Therefore, this study incorporates personality traits to the theoretical model to provide a more thorough understanding of why people are concerned about privacy and thus may decide to disengage in activities that threaten privacy. According to trait theory, a personality trait is useful to help predict individual differences in several behaviors because a personality trait has a tendency to show consistent patterns of thinking, feeling, and behaving (Liu and Amett 2002). This study particularly focuses on narcissism as a novel personality trait to evaluate.

\section{Narcissism}

Personality traits such as narcissism are key factors that may explain how communication works on SNS (Bergman et al. 2011; Carpenter 2012; DeWall et al. 2011; Mehdizadeh 2010; Utz and Kramer 2009). Narcissism is explained with the terms egotistic and arrogant. People who display narcissism are often self-confident and self-absorbed (Mayo Clinic Staff 2011). They tend to boast and brag about themselves to others (DeWall et al. 2011; Mehdizadeh 2010).

Recently, a number of studies have suggested that narcissism is a significant factor driving more people to expose their personal information on SNS (Bergman et al. 2011; Carpenter 2012; DeWall et al. 2011; Mehdizadeh 2010; Utz and Kramer 2009). For example, 
Utz and Kramer (2009) suggested that narcissistic traits lead to less restrictive privacy of personal information on SNS based on research gathered regarding privacy settings of SNS users. Similarly, DeWall et al. (2011) showed that people with narcissistic tendencies communicate on SNS with self-promoting images and words about themselves to promote and draw more attention to them. Carpenter (2012) also argued that narcissistic traits play a role in how SNS are used for individual needs. For example, people who display narcissistic tendency to feel entitlement and to crave attention may use SNS as a means for seeking a large audience for attention by posting more information (Carpenter 2012).

While existing literature seeks to explain the behavioral intentions of SNS users, it is still unknown why people act certain ways (i.e., whether people tend to engage or disengage from SNS activity in different situations), or present themselves in a certain light on SNS (i.e., if people include highly boastful or positive information versus little to no personal information about one's self). Users appear to disclose detailed information about themselves on public profiles which are able to be viewed by other SNS users (Utz and Kramer 2009). This is commonly known as a narcissistic tendency, but ironically those individuals also appear to reveal online privacy concerns about personal information (Utz and Kramer 2009).

Previous SNS studies have approached the concept of narcissism as a one-dimensional concept (Bergman et al. 2011; DeWall et al. 2011; Mehdizadeh 2010). Recent developments in narcissism research, however, suggest there are two subtypes of narcissism that each display unique characteristics (Wink 1991). This suggestion supports that narcissism should be evaluated as a two-dimensional concept most commonly broken into two subtypes: grandiose narcissism and vulnerable narcissism (Hendin and Cheek 1997; Miller et al. 2011). People who display the traits of grandiose narcissism reflect ideals which are referred to as grand, often showing 
aggression and dominate features within their actions (Miller et al. 2011). On the contrary, people who show traits of vulnerable narcissism often reflect, "a defensive and insecure grandiosity that obscures feelings of inadequacy, incompetence, and negative affect" (Miller et al. 2011, p. 1013). Understanding narcissism's subcategories, individually, thus creates a better understanding of each form the personality trait can take on because these two have substantial heterogeneity within the concept of narcissism.

\section{Grandiose vs. Vulnerable Narcissism}

Grandiose narcissism is associated with immediate expression of exhibitionism, selfimportance, and often causes people to be preoccupied with a want for attention from others (Wink 1991). This construct of narcissism primarily reflects traits related to dominate features and aggression which are grand (Miller et al. 2011). Grandiose narcissism is also known as overt (Besser and Priel 2010; Miller et al. 2011).

On the contrary, vulnerable narcissists constantly take notice of criticism and potential failures (Miller et al. 2011). People with vulnerable narcissistic tendencies display traits of defensiveness and insecurity. These traits differ from grandiose narcissism which exhibits traits related to extraversion, self-assurance, aggression and dominance (Wink 1991; Miller et al. 2011). In addition, people who display vulnerable narcissism have feelings of incompetence and inadequacy (Miller et al. 2011). People who show traits of vulnerable narcissism have been described as, "inhibited, shame-ridden, and hypersensitive shy type, whose low tolerance for attention from others and hyper vigilant readiness for criticism or failure makes him/her more socially passive" (Ronningstam 2009, p. 113). Because vulnerable narcissists strive to be vigilant of criticism or potential failures (Miller et al. 2011), they tend to be socially passive and become vulnerable in social situations, specifically when they are unaware of their attention drawing 
ability. These situations ultimately make people with this personality trait vulnerable to the opportunity of interpersonal rejection by peers.

Vulnerable narcissism has taken on multiple terms over the years. Other names for this subcategory of narcissism include: hypersensitive narcissism (Hendin and Cheek 1997) and closet narcissism (Masterson 1993). Research suggests that vulnerable narcissists experience an increased level of anxiety when developing relationships with other people. This means they are often hyper vigilant to separation cues — therefore creating a high experience of distress due to the sensitive nature of their self-esteem (Besser and Priel 2010).

Since people with vulnerable narcissism often feel defensive and insecure, they would be more likely to avoid situations that threaten their personal privacy (e.g., SNS collecting personal information) because of personal fear and insecurity (Miller et al. 2011). Therefore, it is expected that vulnerable narcissism and the likelihood to disengage with privacy-threatening activities on SNS usage have a positive relationship. On the other hand, people with grandiose narcissism would not avoid SNS for this reason. Instead, grandiose narcissists act in a more self-promoting manner because people with the grandiose narcissism trait are often aggressive and dominant; therefore, they would be less likely to remove themselves from the opportunity for dominate and aggressive self-promotion.

Research conducted about narcissism and behaviors on Facebook suggested that there is a positive correlation between people who scored high on the grandiose narcissism evaluation and the amount that they posted on Facebook (Mehdizadeh 2010). Mehdizadeh's study (2010) suggested high narcissism scores have a positive impact on the amount of self-promotional content and the amount of times Facebook was checked by users. Hence, unlike vulnerable narcissism, which has a positive effect on one's likelihood to disengage with privacy-threatening 
activities on SNS, grandiose narcissism may not have a strong influence on one's intention to avoid events potentially threatening his or his privacy. This is because people high in grandiose narcissism may take an active role in seeking self-promoting opportunities, thereby being less vigilant and sensitive about spreading information about themselves on SNS (vs. people high in vulnerable narcissism). Given the distinctive nature of the two types of narcissism, the following hypothesis is developed:

H1: Two types of narcissism will have different influence on consumers' privacy concerns over SNS. More specifically, vulnerable narcissism will have a stronger influence than grandiose narcissism on consumers' likelihood to disengage with privacythreatening activities on SNS usage.

In addition to narcissism, this study investigates other factors that have been identified as significant predictors in privacy literature. These include self-esteem, computer anxiety, and concern for information privacy.

\section{Self-Esteem}

Previous research explains that self-esteem can be a negative predicting factor of several anti-social behaviors on SNS (Carpenter 2012). "Self-esteem is the sense of personal worth and competence that a person associates with his or her self-concept" (Corsini 1984, p. 289). Selfesteem appears to be an important factor to evaluate in this study because previous research has suggested that it is indicative of fundamental differences between the two narcissism constructs (grandiose vs. vulnerable) (Miller et al. 2011). Understanding the role of self-esteem will help bridge a gap in research, as well as act as a potential predicting factor for perceived behaviors. 
Research suggested that individuals with low self-esteem tend to use SNS as a selfpromotional tool (Mehdizadeh 2010). Because of the suggested negative relationship between self-esteem and self-promotion on SNS, it is expected that people with high self-esteem are less likely to need SNS as a means for self-promotion. People with high self-esteem (vs. people with low self-esteem) also tend to be more vigilant when sharing personal information with others online, because they are more concerned with how others evaluate them (Chen et al. 2008). Chen et al. (2008) further argued that people with high self-esteem are more likely to exercise privacy rights since they tend to be obedient to the standard of social mortality to maintain their individual reputation. As a whole, it suggested that people with high self-esteem will be more likely to avoid harmful events potentially threatening their privacy because they are motivated to protect their reputation, as well as to meet the standard of social mortality. It is therefore hypothesized that self-esteem and one's likelihood to disengage with privacy-threatening activities on SNS usage will have a positive relationship. Thus, the following hypothesis is developed:

H2: Self-esteem will have a positive influence on consumers' likelihood to disengage with privacy-threatening activities on SNS usage.

\section{Computer Anxiety}

Korzaan and Boswell (2008) explain that the anxieties of online use influence the overall opinion of privacy infringement in the eyes of the user. Furthermore, personality traits have been found to have a strong basis to explain computer anxiety (Korzaan and Boswell 2008). While computers have become a facet of everyday life for many people in the western-world, they have been found to pose a specific form of anxiety for some individuals who have a strong fear of the 
technology (Beckers and Schmidt 2003). This fear, also known as computer anxiety, can be experienced in various forms and to various degrees of extent.

Two types of anxiety traditionally exist: state anxiety and trait anxiety. State anxiety is created by a current situation whereas trait anxiety is caused by a dispositional anxiety that is normally experienced by people who often worry or fear of failing in a given situation (Parayitam, Desai and Eason 2010). The present study focuses on trait anxiety to help explain individual differences. Increased levels of anxiety have been reported to lead to negative physiological arousal as well as inhibited thoughts (Heinssen, Glass and Knight 1987). Individuals with a high level of computer anxiety who experience arousal and inhibited thought are less likely to behave comfortably around computers than do individuals whose level is high (Stewart and Segars 2002). Hence, it is expected that consumers who exhibit a high level of computer anxiety are more likely to remove their personal information from SNS and refuse to provide it in the future. In fact, a number of studies have suggested that computer anxiety is highly related with consumer concerns over privacy issues on the Internet. For example, Stewart and Segars (2002) showed that computer anxiety positively influences behavioral intention to engage in privacy-protecting activities. Korzaan and Boswell (2008) also suggested that individuals who have a high level of computer anxiety display a high level of privacy concerns over the Internet, given inherent distrust of the technology among those who have a high computer anxiety. The positive relationship between computer anxiety and the likelihood of disengaging privacy-threatening activities on SNS usage is thus expected.

H3: Computer anxiety will have a positive influence on consumers' likelihood to disengage with privacy-threatening activities on SNS usage. 


\section{Concern for Information Privacy (CFIP)}

Freely exchanging information online often brings threats upon consumers due to providing easy, and often, unwanted, access to personal information (Stewart and Segars 2002). While SNS information privacy concern is a specialized entity, recent research on general information privacy is also able to be applied to the concept because of cross-avenues of research application. Previous research on attitudes regarding information privacy many times addresses the perceptions of policy practices in effect for information privacy (Bélanger and Crossler 2011).

The concept of concern for information privacy has recently received a great deal of attention among information systems scholars. The term, concern for information privacy (CFIP), was coined by Smith et al. (1996). The term refers to consumers' concern about how organizations use and protect their information privacy. All online interactions demand a level of trust to build security between the users and the Internet-based business (Chen and Rea, 2004). According to Smith et al. (1996), consumers have four different responses to organizations' information privacy practices: collection, unauthorized secondary use, improper access, and errors.

Collection refers to the concern over whether data are collected and stored appropriately. Unauthorized secondary use involves concerns regarding whether data collected for a certain purpose will be inappropriately used for someone else without authorization. Third, improper access reflects to consumers' concerns over whether unauthorized people can view their personal information. Finally, error defines consumers' concerns about whether their personal data will be adequately protected against accidental or intentional errors (Smith et al. 1996). 
Stewart and Segars (2002) further empirically tested the concept of CFIP, and provided a validated CFIP measurement model with multiple dimensions. They also found that CFIP mediated a relationship between computer anxiety and consumers' behavioral intention to retaliate against company's practices of collecting personal data. Recently, Korzaan and Boswell (2008) also suggested that CFIP improves the predictability of personality traits on behavioral intention. In details, they suggested the impact of five-factor personality trait on behavioral intention regarding privacy issues is determined by consumers' CFIP.

While SNS information privacy concern is a specialized entity, recent research on CFIP applies to SNS because of shared characteristics of Internet across diverse venues. For example, continuous changes in posts on SNS advance concerns for security because individuals are not always able to keep up with the real-time information and photo updates. Therefore, it is hypothesized that there would be a positive correlation between CFIP and likelihood to disengage with privacy-threatening activities on SNS usage.

H4: Concern for information privacy (CFIP) will have a positive influence on consumers' likelihood to disengage with privacy-threatening activities on SNS usage. 


\section{CHAPTER 3. STUDY I}

\section{Overview and Design}

Study I examines psychological factors, more specifically narcissism — as a dual-faceted construct — that predict one's likelihood to disengage with privacy-threatening activities on SNS usage. A survey was administered to evaluate individual's vulnerable and grandiose narcissism measurement, as well as their self-esteem, computer anxiety, concern for information privacy, and likelihood to disengage from privacy-threatening activities on SNS usage. To examine the extent to which one's psychological factors influence his/her likelihood to disengage from privacy-threatening activity on SNS usage, a step-wise regression analysis was conducted.

\section{Method}

\section{Sample}

A total of 176 undergraduate students participated in the study in exchange for extra course credit. Females comprised $55.7 \%$ of the sample and the average age of the sample was 20.5 years. The study was completed as a self-administered, web-based survey.

\section{Measures}

Grandiose narcissism was measured with a short pair measure created by Ames, Rose and Anderson's study (2006). The grandiose narcissism measure includes 16 items such as "I can make anybody believe anything I want them to" (Ames, Rose and Anderson 2006). Vulnerable narcissism was measured with a 10-item measurement adopted by Hendin and Cheek (1997). Measurement items include the statement such as, "My feelings are easily hurt by ridicule or by the slighting remarks of others" (Hendin and Cheek 1997). Appendix 1 includes grandiose and vulnerable narcissism measures. 
The measurement of computer anxiety was adopted from Stewart and Segars (2002). It includes four items such as "Computers are a real threat to privacy in this country" (Stewart and Segars 2002). Measuring self-esteem was accomplished with the Rosenberg Self-Esteem Scale. The scale was composed of 10 items. Sample items include: "I feel that I'm a person of worth, at least on an equal plane with others" (Rosenberg 1965). Concern for information privacy (CFIP) was evaluated with 15 items. This evaluation was adopted from Smith et al.'s study (1996). Sample items include: "It bothers me to give personal information to so many companies" (Smith et al. 1996). All items except grandiose narcissism were measured using seven-point agreement scales, where the points were labeled "strongly disagree," "disagree," "somewhat disagree," "neither agree nor disagree," " somewhat agree," "agree," and "strongly agree." Appendix 1 includes computer anxiety, self-esteem, and concern for information privacy measures.

Finally, the likelihood to disengage with privacy-threatening activities on SNS usage was measured with a modified version of questions developed by Stewart and Segars (2002). The statements were evaluated on a seven-point Likert-type scale: $(1)=$ "Very Unlikely" and (7) = "Very Likely." An example of an item in this section was: "How likely are you within the next three years to ... Take action to have your name or photos of you removed or placed under more strict privacy settings on social networking sites?” Appendix 1 includes behavioral intention measures.

\section{Results}

Before the main analysis was conducted, multicollinearity analysis was conducted to confirm whether each construct, specifically vulnerable and grandiose narcissism, was orthogonal from each other. Since multicollinearity exists when a tolerance is less than .10 and 
variance inflation factor (VIF) is greater than 5 (O'Brien 2007), there is no multicollinearity for any of the constructs explored in this study. Table 1 reports multicollinearity test results.

Table 1

Multicollinearity Analysis

\begin{tabular}{lcc}
\hline Measure & Tolerance & VIF \\
\hline Grandiose Narcissism & .84 & 1.18 \\
Vulnerable Narcissism & .65 & 1.52 \\
Self-Esteem & .77 & 1.29 \\
Computer Anxiety & .68 & 1.47 \\
Concern For Information Privacy & .76 & 1.31 \\
\hline
\end{tabular}

Prior to testing the hypotheses, descriptive statistics were run to examine the personality traits dispersion among the college student participants. Table 2 displays the descriptive statistics of each measure along with the reliability coefficients.

Table 2

Study I Means, standard deviation, scale reliability

\begin{tabular}{lccc}
\hline \multicolumn{1}{c}{ Measure } & $\boldsymbol{M}$ & $\boldsymbol{S D}$ & Reliability \\
\hline Grandiose Narcissism & 1.61 & .21 & .72 \\
Vulnerable Narcissism & 3.72 & .90 & .78 \\
Self-Esteem & 5.38 & 1.08 & .89 \\
Computer Anxiety & 3.97 & 1.10 & .75 \\
Concern for Information Privacy & 5.17 & 1.26 & .96 \\
Behavioral Intentions* & 4.34 & 1.36 & .83
\end{tabular}

* Behavioral Intentions represent the likelihood to disengage with privacy-threatening activities on SNS usage.

A two-step, step-wise regression was conducted to clarify whether there was additional variance between the constructs. The likelihood to disengage with privacy-threatening activities on SNS usage was used as the dependent variable for the analysis while self-esteem, computer 
anxiety, and CFIP remained constant. In step-one $r^{2}=.217$. Once vulnerable and grandiose narcissism were added in step-two, $r^{2}=.259$ accounting for a $\Delta r^{2}=.042$. Thus, adding narcissism constructs increases the predictability of consumers' response to organizational practices of collecting personal information on SNS.

As displayed in table 3, grandiose narcissism was not a significant predictor of the likelihood to disengage with privacy-threatening activities on SNS usage $(\beta=.11, p=.09)$ while vulnerable narcissism had a significant influence on the likelihood to disengage with privacythreatening activities on SNS usage $(\beta=.23, p<.01)$. Therefore, hypothesis 1 is supported.

As for self-esteem $(\beta=.19, p<.01)$, it appeared to be a significant predictor of the likelihood to disengage with privacy-threatening activities on SNS usage, thereby supporting hypothesis 2 . Likewise, computer anxiety $(\beta=.23, p<.01)$ and $\operatorname{CFIP}(\beta=.18, p<.05)$, appeared to be significant predictors. Therefore, hypothesis 3 and hypothesis 4 were supported.

Table 3

Multiple Regression Step-Wise Method Analysis

\begin{tabular}{lll}
\hline Variables & Step $\mathbf{~}$ & Step 2 \\
& $\boldsymbol{B}$ & $\boldsymbol{B}$ \\
\hline Self-Esteem & .097 & $.194^{* *}$ \\
Computer Anxiety & $.329 * *$ & $.234^{* *}$ \\
Concern For Information Privacy & $.211^{* *}$ & $.185^{*}$ \\
Grandiose Narcissism & & .118 \\
Vulnerable Narcissism & & $.234^{* *}$ \\
& $R^{2}=.217$ & $\Delta R^{2}=.042$ \\
& $F(3,172)=15.915$, & $F(2,170)=4.795$, \\
& $p<.001$ & $p<.009$ \\
\hline
\end{tabular}

Note: Beta-weights marked with "*” are significant at the $p<.05$ or greater. Beta-weights marked with "*** are significant at the $\mathrm{p}<.01$ or greater. 


\section{Discussion}

The purpose of Study I was to explore distinctive relationships between two types of narcissistic traits and the likelihood to disengage with privacy-threatening activities on SNS usage while also looking at the effects of previous known variables (i.e., computer anxiety, selfesteem, and concern for information privacy). The results of Study I show that vulnerable narcissism has a significant influence on consumers' likelihood of disengaging with privacythreatening activities on SNS while grandiose narcissism has no influence. Previous studies demonstrate a significant positive correlation between people who scored high on grandiose narcissism and general SNS activity (Mehdizadeh 2010). The current findings suggest that there may be a moderating impact that has not been accounted for in previous literature on SNS. No significant relationship between grandiose narcissism and the likelihood to disengage with privacy-threatening activities on SNS usage (vs. the significant effect of vulnerable narcissism) suggests that narcissism as a whole cannot solely be a predicting source of behavior, but instead must be broken down into constructs (i.e., grandiose and vulnerable) to better understand traits people have which may influence their likelihood to prevent privacy-threatening activities on SNS usage.

Consistent with prior studies, results provided a positive relationship between selfesteem, computer anxiety, and CFIP with one's likelihood to disengage with privacy-threatening activities on SNS. Results show that individuals with high computer anxiety or CFIP are each more likely to disengage with privacy-threatening SNS activity. The outcomes were consistent with prior studies. Thus, results indicate that individual differences in self-esteem, computer anxiety, and CFIP are important variables that can affect consumers' responses to companies' practices of collecting personal information on SNS. A positive correlation was also found 
between self-esteem and concern for information privacy. This is supported by a Pearson Correlation which revealed a significant correlation between these two constructs (Pearson Correlation $=.204, p<.01, N=176)$. To better understand the actual causation of the constructs further research would need to be developed. 


\section{CHAPTER 4. STUDY II}

Study I proposed and tested the role psychological factors play in predicting one's likelihood to disengage with privacy-threatening activities on SNS usage by using a twodimensional approach of narcissism. More specifically, Study I provided that vulnerable narcissism has a highly significant, positive relationship with one's response to privacythreatening activities on SNS whereas grandiose narcissism has no impact. The findings of Study I suggested that SNS researchers should move beyond the current research tradition of a onedimensional approach to narcissism in the evaluation process of SNS usage in order to benefit from a redirection by incorporating a two-dimensional approach of narcissism.

To advance our understanding of how specific subcategories of narcissism impact the way people intend to behave on SNS, Study II was developed to evaluate whether the link between vulnerable narcissism and one's likelihood to disengage with privacy-threatening activities on SNS differ in terms of reactions to threats involving interpersonal rejection.

\section{Interpersonal Rejection}

People in general are social and have an innate longing to belong with others (Leary 2001). This longing to belong to others goes so far that most individuals even fear the potential rejection of other people. Particularly, narcissistic individuals are sensitive and reactive to the experience of their relationship partner's rejection. Within the context of interpersonal relationships, narcissists commonly use their relationships with significant others as a way to regulate and maintain a positive self-image (DeWall et al. 2011). Because of the consequences that rejection has on people, it is very common for humans to create mechanisms which actually help them avoid fear and hurt which comes with rejection and in return actually avoid the interpersonal rejection all together (Leary 2001). This avoidance is often reflected when people 
work to protect personal information by showing concern for privacy to ultimately avoid potential catalysts for rejection.

Recent developments in narcissism literature have suggested that grandiose narcissists and vulnerable narcissists respond differently to interpersonal rejections. For example, Besser and Priel (2010) suggested that high vulnerable narcissistic people (vs. high grandiose narcissistic people) tend to display higher stress levels when they experience threats involving a romantic partner's rejection. This is because threatening situations vary in their pertinence to vulnerable narcissism versus grandiose narcissism (Besser and Priel 2010).

While few studies have been conducted to relate vulnerable narcissism to rejection sensitivity, the concepts are described very similarly as concepts of psychological insecurity (Hendin and Cheek 1997). The similarities suggest potential parallels in behavioral reactions to interpersonal social rejection. For example, people who are sensitive to social rejection tend to overreact; therefore, it is hypothesized that people with high levels of vulnerable narcissism will have a heightened likelihood to disengage with privacy-threatening activities on SNS usage when introduced to high interpersonal rejection threats.

In this line, the continuous search for approval and constant need for validation of peers and other people to maintain an enhanced self-esteem level is associated with a consistent pattern for vulnerability in narcissists (Besser and Priel 2010). Furthermore, previous research explains that self-esteem can be a negative predicting factor of several anti-social behaviors on SNS (Carpenter 2012). The need for vulnerable narcissists to uphold a personal sense of self-worth maintains the idea that people with vulnerable narcissistic tendencies are likely to disengage from SNS usage when their sense of self-esteem may be threatened by the lack of validation from others. 
In addition, people who are narcissistic use strategic mechanisms to communicate with others in a way that allows them to preserve positive attention on themselves (DeWall et al. 2011). A disturbance in this strategy could alter one's behavior and make him or her more likely to disassociate with privacy-threatening activities on SNS to avoid the dissonance created by a rejection threat. Study I revealed a significant positive correlation between vulnerable narcissism and the perceived likelihood to disengage with privacy-threatening activities on SNS usage. Hence, it is argued that people with high vulnerable narcissism (vs. low vulnerable narcissism) will be more likely to disengage with privacy-threatening activities on SNS usage when they are introduced to a high interpersonal rejection threat (vs. low interpersonal rejection threat). It is anticipated that the effect of vulnerable narcissism would be more robust under conditions of high interpersonal rejection. In light of these considerations the following hypothesis has been formulated:

H5: The relationship between vulnerable narcissism and likelihood to disengage with privacy-threatening activities on SNS usage will be stronger when individuals are introduced to high interpersonal rejection threats (versus low interpersonal rejection threats).

\section{Method}

\section{Overview and Design}

Study II re-examines psychological factors that predict one's likelihood to disengage with privacy-threatening activities on SNS usage. Study II expands on Study I with studying the effect interpersonal rejection threats have on SNS usage behavior. A quasi-experiment was designed to test the hypothesis. A quasi-experimental model was used because arbitrarily randomizing 
personality traits (e.g., using a median-split to label the people above the median as high vulnerable narcissists, and those below the median as low vulnerable narcissists) may not be practical and statistically accurate. As Cohen (1983) argued, breaking subjects into two groups leads to the loss of $1 / 5$ to $2 / 3$ of the variance accounted for by the original variables. Thus, the level of interpersonal rejection was manipulated while vulnerable narcissism was measured and treated as a continuous variable in the quasi-experiment setting.

\section{Sample}

A total of 181 undergraduate students participated in the study in exchange for extra course credit. All participants were required to be at least 18 years of age or older. Females comprised $46.4 \%$ of the sample and the average age of the sample was 20 years old. The study was completed as a self-administered, web-based questionnaire to complete a quasi-experiment.

\section{Stimuli Development and Pre-test}

To provide a meaningful examination of the hypotheses, the scenarios of high and low threats of interpersonal rejection on SNS were developed based on Besser and Priel's (2010) study (Appendix 2). The high-level threat scenario introduced a situation where the person logs onto a SNS and finds out a friend on SNS is no longer connected (e.g., Friend on Facebook or Twitter) to them while the low-level threat scenario introduced a situation where the person logs onto a SNS and believes a SNS friend is upset with them because of a statement posted on the SNS but later finds out the friend was actually upset about an unrelated issue. Participants were requested to assume the role of the person described in the scenario and to indicate the degree to which the threats would appeal to their personal interests for assuring the manipulation.

Further, a pre-test was conducted on a total of 79 undergraduate students to confirm the manipulation effects before conducting the main study. All participants were required to be at 
least 18 years of age or older. Females composed $49 \%$ of the sample and the average age of the sample was 22 years. Participants were randomly assigned in two conditions with a high or low threat version. They were asked to read a brief scenario and rate the extent to which the scenario threatened them and to answer the extent to which the scenario made them angry. The pre-test result showed a significant difference for perceived threat between the high and low threat scenarios $\left(M_{\text {High }}=3.54, S D_{\text {High }}=1.85\right.$ vs. $\left.M_{\text {Low }}=2.37, S D_{\text {Low }}=1.44 ; t(79)=3.15, p=.002\right)$. Thus, the scenarios were chosen to manipulate the interpersonal rejection experimentally.

\section{Measures and Procedure}

Two questionnaire versions - A and B - were used, each with a hypothetical scenario to read with the intention of evoking the threat of high or low levels of interpersonal rejection. SNS usage was measured with a five-item measurement adopted from Bowman, Westerman, and Claus (2012) (Appendix 1). The questions evaluated individual's amount, frequency, and duration of time spent on SNS. Amount was measured on a six-point scale ranging from " 1 = I do not use social networking sites" to " $6=$ more than 6 hours." Frequency was measured on a six-point scale ranging " $1=$ none" to " $6=30$ times or more per day." Duration was also measured on a six-point scale and ranged from " $1=$ less than 1 year" to " $6=$ more than 5 years." The fourth question evaluated how individuals evaluated themselves (i.e., user type) ranging from " 1 = light user" to " $4=$ I do not use Social Networking Sites." The last question was openended and asked for participants to briefly describe their reason for using SNS.

The questionnaire set for the vulnerable narcissism was measured with a 10-item measurement adopted by Hendin and Cheek (1997). Similar to Study I, the grandiose narcissism measure included 16 items as a short pair measure created by Ames et al.'s study (2006). During the scenario, high and low interpersonal rejection threat was introduced with a modified version 
of scenarios developed by Besser and Priel (2010). Participants were requested to assume the role of the person described in the scenario and to indicate the degree to which the threats would appeal to their personal interests for assuring the manipulation. The first set of questions on of the manipulation check was measured on a seven-point scale ranging from " $1=$ not at all" to "7 = very much" and was adopted from Besser and Priel (2010). It was composed of three questions including: To what extent do you feel threatened by the situation above?" The second set of manipulation questions evaluated anger responses using questions from the State Anger Scale (STAS) developed by Spielberger et al. (1983). It was composed of three questions including: "I feel angry" (Spielberger et. al 1983) and evaluated on a seven-point scale ranging from " $1=$ not at all" to "7 = very much." The final set of manipulation questions evaluated current, negative state mood. Negative state mood was measured with a modified version of the Visual Analogue Scales (VAS) adopted by Abersnagel (1988) and Besser and Priel (2009) used to measure momentary moods. The measurement includes assessments of anxious states. The scale was composed of 4 negative mood adjectives that participants were asked to evaluate ranging from " 1 $=$ not at all" to "7 = very much." The state anger and the negative state mood were used because anger may be one outlet for individuals to alleviate tension created by rejection and because anxiety over abandonment may increase negative mood state (Besser and Priel 2009).

For the dependent variable, the likelihood to disengage with privacy-threatening activities on SNS was measured with a modified version of questions developed by Stewart and Segars (2002). 


\section{Results}

\section{Manipulation Checks}

To ensure the interpersonal rejection manipulation successfully induced a threat, participants were asked to complete a series of manipulation check questions. Independent sample t-tests were conducted to compare the manipulation check in the high and low threat scenario conditions. For the questions asking perceived threat levels, there was a significant difference in the scores for the high-threat and low-threat conditions $\left(M_{\mathrm{HIGH}}=4.31, S D_{\mathrm{HIGH}}=1.55\right.$ vs. $M$ Low $\left.=2.48, S D_{\text {Low }}=1.42 ; t(181)=8.24, p<.001\right)$. Anger states also displayed a significant difference in scores for the high threat and low threat conditions $\left(M_{\mathrm{HIGH}}=4.08, S D_{\mathrm{HIGH}}=1.79\right.$ vs. $\left.M_{\text {Low }}=2.03, S D_{\text {Low }}=1.26 ; t(181)=8.86, p<.001\right)$. The same line followed for the negative mood state which showed a significant different in scores for the high threat and low threat conditions $\left(M_{\text {HIGH }}=3.86, S D_{\text {HIGH }}=2.00\right.$ vs. $M_{\text {LOw, }} S D_{\text {LOW }}=1.53=2.37 ; t(181)=5.61, p<.001$. Thus, the manipulation was highly significant. Table 4 summarizes the results of manipulation.

Table 4

The Effectiveness of Study II Manipulation

\begin{tabular}{lccccc} 
& \multicolumn{1}{c}{ High } & & Low & & \\
& Mean & SD & Mean & SD & p-Value \\
\hline Perceived Threat & 4.31 & 1.55 & 2.48 & 1.42 & $<.001$ \\
Anger States & 4.08 & 1.79 & 2.03 & 1.26 & $<.001$ \\
Negative Mood States & 3.86 & 2.00 & 2.37 & 1.53 & $<.001$ \\
\hline
\end{tabular}




\section{Descriptive Statistics}

Prior to testing the hypothesis, descriptive statistics were run to examine the variable dispersion among participants. Table 5 displays the descriptive statistics of each measure along with the reliability coefficients.

\section{Table 5}

\section{Study II Means, Standard Deviation, and Scale Reliability}

\begin{tabular}{lccc}
\hline \multicolumn{1}{c}{ Measure } & $\boldsymbol{M}$ & $\boldsymbol{S D}$ & Reliability \\
\hline Grandiose Narcissism & 1.63 & .22 & .76 \\
Vulnerable Narcissism & 3.63 & .78 & .67 \\
Computer Anxiety & 3.71 & 1.15 & .75 \\
Self-Esteem & 5.44 & 1.12 & .91 \\
Concern for Information Privacy & 5.13 & 1.10 & .92 \\
Behavioral Intentions* & 3.59 & 1.51 & .84 \\
Manipulation Check & 3.40 & 1.75 & .88 \\
State Anger & 3.06 & 1.86 & .97 \\
Negative Mood State & 3.13 & 1.93 & .95 \\
*Behavioral Intentions represent the likelihood to disengage with privacy-threatening \\
activities on SNS usage.
\end{tabular}

Descriptive statistics were also run to examine the general use of SNS among the participants. Results show that of the participants, 32\% are on SNS 1-2 hours daily, while another $29.1 \%$ engage in SNS activity 3-4 hours a day. Additionally, $61.3 \%$ of the 181 participants check SNS 10 or more times daily (28.7\% check 10-19 times daily, $16 \%$ check 2029 times daily, and $16.6 \%$ check 30 times or more daily). $38.7 \%$ of participants estimate that their usage pattern has existed for four years or more. Meanwhile, $82.4 \%$ of participants describe themselves as medium or heavy SNS users (59.7\% medium users, $22.7 \%$ heavy users). Table 6 summarizes the results of SNS usage. 
Table 6

Study II SNS Usage Means and Standard Deviation

\begin{tabular}{lcc}
\hline \multicolumn{1}{c}{ Measure } & $\boldsymbol{M}$ & $\boldsymbol{S D}$ \\
\hline Amount & 3.69 & 1.19 \\
Frequency & 3.97 & 1.29 \\
Duration & 3.78 & 1.64 \\
User Type & 2.05 & .64 \\
\hline
\end{tabular}

\section{Hypothesis Testing}

To test the hypothesis, a multiple regression was conducted. Table 7 describes the results of the multiple regression analysis. Inconsistent with the results of Study I, a multiple regression analysis revealed that vulnerable narcissism had no influence on likelihood to disengage in privacy-threatening activities in SNS.

Table 7

Multiple Regression Analysis of Variables

\begin{tabular}{lccl}
\hline \multicolumn{1}{c}{ Measure } & $\boldsymbol{B}$ & $\boldsymbol{T}$ & $\boldsymbol{p}$ \\
\hline Grandiose Narcissism & -.094 & -1.298 & .196 \\
Vulnerable Narcissism & -.112 & -1.328 & .186 \\
Self-Esteem & -.112 & -1.409 & .161 \\
Computer Anxiety & .151 & 1.798 & .074 \\
Concern for Information Privacy & .265 & 3.365 & $.001^{* *}$ \\
\hline
\end{tabular}

Note: $* * \mathrm{p}<.01$

Given the lack of significant results in Study II, it was determined that further statistical analysis was unnecessary, thereby not confirming H5. No step-wise regression analysis was conducted for Study II because the multiple regression analysis did not show an influence on the likelihood to disengage with privacy-threatening activity on SNS. Given this result, the comparison made in a step-wise function would not reveal additional information. 


\section{Discussion}

The purpose of Study II was to advance the findings in Study I by re-evaluating the role of narcissism in predicting one's likelihood to disengage with privacy-threatening activities on SNS. Particularly, Study II evaluated the likelihood of SNS users who display the vulnerable narcissism trait to disengage with privacy-threatening activities on SNS when introduced to threats (high vs. low) involving interpersonal rejection. Study II also sought to understand if interpersonal rejection threat acts as a moderating link with vulnerable narcissism and the other outcome variables (self-esteem, computer anxiety, and concern for information privacy).

The results of Study II revealed that vulnerable narcissism does not influence how people respond to privacy threatening activities in SNS, thereby not confirming the link identified in Study I. To understand this unexpected result, further investigation into the patterns of negative emotional states and anger expression across two threat conditions was performed. The results showed that people with high vulnerable narcissism were more likely to display negative emotional states and express anger than those with low vulnerable narcissism. This finding is consistent with Besser and Priel's study (2010). The result indicates that when people experienced interpersonal rejection on SNS, vulnerable narcissism influences how they perceive threats involving interpersonal rejection but not necessarily how they respond to privacy issues on SNS. Inoculation theory (McGuire 1964) may provide some insights into this interesting result.

William McGuire developed inoculation theory in the 1960s (Ivanov, Pfau, and Parker 2009; Pfau 2007). Inoculation theory postulates that individuals can be inoculated against future attitude attacks much the same way individuals can be inoculated against future viral attacks. Just like a biological inculcation allows a human to build resistance against future viral attack, an 
attitudinal inoculation warns an individual of an attitudinal attack against an existing attitude (McGuire 1964). The theory is used, "as a strategy to protect attitudes from change — to confer resistance to counter attitudinal influences, whether such influences take the form of direct attacks or sustained pressures" (Pfau 2007). Inoculation theory is composed of two key elements: threat and refutational preemption (Pfau 2007). Threat is the component that acts as a motivational source to resistance because it causes individuals to notice the potential for existing attitudes to be modified (Pfau 2007). The refutational preemption is content that can be used by people to defend existing attitudes because it raises arguments contradictory to attitudes and then refutes them. Together, these components make inoculation theory work through the use of threats and counterarguments (Pfau 2007).

According to the inoculation theory, elements of threat may generate the acknowledgement that an attitude is vulnerable; however, the acknowledgement often induces a motivation for individuals to strengthen their pre-existing attitude (e.g., their attitude prior to being threatened) (Ivanov, Pfau, and Parker, 2009). What this suggests for Study II, is that SNS study participants have virtually become immune to certain dosages of social rejection on SNS because they are exposed to it in small amounts (i.e., inoculated by the threat scenario). However, given the amount of SNS usage participants engage in (based on the SNS usage data collected in Study II), the act of using SNS outweighs the likelihood to disengage with SNS usage because of one threatening instance. Therefore, presumably when introduced to an interpersonal rejection threat in Study II, participants may acknowledge the threat made them vulnerable (i.e., the high success of the manipulation), but then strengthen their pre-existing behavior of using SNS despite the privacy risks. That is, interpersonal rejection threats used in Study II may provide specific content that people can employ to strengthen attitudes against 
subsequent change (e.g., protecting their privacy by disengaging from activities where they would provide personal information on SNS.)

Additionally, Leary (2001) explains that people create mechanisms to avoid the fear and rejection presented with interpersonal rejection threats. This further supports the inoculation theory as a mechanism that helps SNS users disregard the rejection, but continue to maintain their prior behaviors. Hence, the interpersonal threat manipulation was successful; however, the inoculation theory mechanism - to avoid the negative feelings - overrides potential modified behavior changes as hypothesized.

The unexpected results may also be linked to the degree of change the step-wise analysis explained in Study I. The results of the step-wise regression showed that vulnerable narcissism increased $R^{2}$ by. 042 . Vulnerable narcissism has predictability but is not a dramatic predictor for the likelihood to disengage with privacy-threatening activity on SNS. Hence, Study II's results may not confirm the link developed in Study I because of the potential for vulnerable narcissism to not be a single predictor of the likelihood to disengage from privacy-threatening activity on SNS.

Another possible explanation for this unexpected finding is that while participants were asked to imagine themselves in the given scenarios that were proximal, the dependent variable (i.e., likelihood to disengage from SNS) questions asked distal intention (e.g., in the next three years how likely are you to...) rather than proximal intention (e.g., right now how likely are you to...). The questions were designed to maintain consistency with Study I questions. However, maintaining consistency within the questionnaire would alleviate altering mental states (e.g., thinking in the present as opposed to thinking into the future). Given that the threat was highly 
successful, the temporal distance of dependent variable questions may influence how participants perceive the threat, as well as form future behavior intentions. 


\section{CHAPTER 5. GENERAL DISCUSSION}

The purpose of this study was to explore distinctive relationships between two types of narcissistic traits - vulnerable and grandiose narcissism - and the likelihood to disengage with privacy-threatening activities on SNS while also examining the effects of previously known variables (i.e., self-esteem, computer anxiety, and concern for information privacy). As one of the first studies to apply a two-dimensional approach to narcissism in communication context, the research contributes an extension to literature on multiple fronts.

First, it shows that narcissism (on a macro-level), as a one-dimensional construct, cannot solely predict the source of behavior, but instead must be broken down into constructs (i.e., grandiose and vulnerable) to better understand the personality trait that influences behaviors. In particular, the result of Study I showed distinctive patterns across two dimensions of narcissism: people who are high in vulnerable narcissism (vs. low) are more likely to disengage with SNS activities when they perceive a threat to their privacy, whereas no relationship exists between grandiose narcissism and the likelihood to disengage with privacy-threatening activities on SNS usage.

The important empirical evidence offered by the data is that narcissism has substantial heterogeneity within the construct, as two forms of narcissism shared little with regard to individual behaviors on SNS. The application of a dual-faceted approach creates a more thorough understanding of how two distinct narcissistic tendencies apply to individuals'

likelihood to disengage with privacy-threatening activities on SNS as a theory. Breaking down narcissism into two constructs gives media psychology researchers a better understanding of how the narcissism personality trait impacts privacy-threatened intentions on SNS. 
However, Study II suggests when people are exposed to interpersonal rejection threats on SNS, the effects of vulnerable narcissism on their intention to disengage with privacythreatening activity are largely attenuated. The result suggests that the theoretical link between vulnerable narcissism and responses to privacy-threatening activities on SNS apply only to the macro level. Study II introduced that on the micro-level, situations where individuals are actually encountering threatening activities on SNS, the theoretical link found in Study I has fallen short in its ability to explain the effects of personality traits on SNS behavioral intention.

Such a finding is particularly interesting because it advocates for the integration of macro-micro theories to the field of communication. Traditionally, communication literature has been dominated by a single, micro-level theoretical perspective. However, inconsistent findings from the two studies indicate that development of multi-level theories is needed to explain macro-micro dynamics in society. By analyzing individual behavior and the social contexts, the move toward an integration of macro-level and micro-level analysis provides a better picture of the phenomenon.

The suggestion of a macro-micro theory contributes to more than theology. The theory is also important for corporate entities. Understanding the macro- and micro-level of SNS behavior changes will help corporations interpret any direct effects of individual and contextual variables (Muftic 2009), more specifically the likelihood to disengage from privacy-threatening activity on SNS. An example of applying this to life would be when a company loses SNS hits (the number of times SNS users view the companies SNS page), data interpretation will develop more than one potential causal explanation. Given a more in-depth explanation gives companies a better frame-work to modify their page based on multiple potential causes of behavior potentials of SNS users. 
Second, Study I contributes to literature on Internet privacy by showing a positive relationship between self-esteem, computer anxiety, CFIP and likelihood to disengage with privacy-threatening activities on SNS. The results provide that individuals with high self-esteem, computer anxiety, or CFIP are each more likely to disengage with privacy-threatening SNS activity. As such, results indicate that individual differences in self-esteem, computer anxiety, and CFIP are important variables that can affect SNS users' responses to companies' practices of collecting personal information on SNS. Given that concerns over privacy are often considered the forefront obstacle to SNS growth (O’Brien and Torres 2012), identifying psychological factors which increase users' likelihood of disengaging with SNS will advance researchers' knowledge about SNS usages.

Managerially, the results could be used by SNS companies and advertising firms to better track and keep up with SNS user maintenance. To keep users from disengaging from SNS, company managers must understand how SNS user personalities affect their usage. Utilizing the theoretical framework set up by this study helps advance research to find how to not only engage users, but more importantly how to help maintain an atmosphere that fosters a more pleasant experience for users if the SNS or its policies are adaptable based on individual's personality traits. For example, it is important that companies clearly communicate their privacy policies and practices with those who are high in vulnerable narcissism because they are hyper vigilant about personal information. Also, new features should be adequately accompanied by several selfprotective functions that allow consumers to protect themselves from identify thefts.

Results from this research study will also impact SNS users. The understanding of privacy threat tendencies based on what is posted on SNS influences people as a whole. By understanding how it influences vulnerable narcissists, it will not only help people understand 
themselves; but also, help people better understand the usage of other SNS users. Creating a more developed understanding of how the communication of SNS works and impacts others will help enable people to create a better environment for the technology-driven communication. This will help foster an environment that could alleviate an issue explained by Ryan, Magro and Sharp (2011) as a sense of "isolation among students" (p. 6). While this contribution cannot actually make the changes for the better environment, it can foster the knowledge to enable the environment to exist.

\section{Limitations of Study}

Although this study advances SNS literature by offering theoretical and managerial contributions, some limitations should be considered when interpreting the results. This study analyzes the behavioral intentions of SNS users but does not account for the actual behaviors of SNS users. Therefore, it serves as a starting point to better understand potential causation for certain behaviors on SNS, but further analysis should be made to understand not only users' perceptions of their potential behaviors but also SNS users' actual behaviors. Second, the generalization of the study is limited by the use of collegiate subjects who each use SNS and were within one area of study. Therefore, future study with a larger sample, containing a balanced mix of all age groups, with participants who do not use SNS, and is drawn from random sampling will be needed to make the results generalizable. Third, the study sample size limits strong analysis of additional potential factors which may play a role in the likelihood to disengage with privacy-threatening activities on SNS usage. For example, this study did not account for gender difference or ethnic differences. Fourth, the manipulation scenario creates an interpersonal rejection threat, but does not seek to address privacy in the scenario. Hence, modification of the scenario may alter the outcome results of the analysis. Fifth, while the 
theoretical pinning of inoculation theory may support the phenomenon present in Study II, it is one avenue to explore and other theories may explain the phenomenon. Finally, questions used in Study II's behavioral intention section of the study address behaviors for the future in an effort to maintain consistency with Study I. A more immediate behavior modification model for the questions may make a difference in the results.

\section{Directions for Future Research}

To enhance the contributions of this study, future research should examine the issue of whether people have accepted SNS privacy attacks because the use of the SNS is more valuable to them than its potential dangers. According to theory of the privacy paradox, Internet users are concerned about privacy yet do little to protect their privacy because the benefits seemingly outweigh the losses (Barnes 2006). Future research should investigate how SNS users overcome privacy anxiety to meet their desire for social interaction.

Continuing research on user privacy will also explain where social media outlets need to move forward with privacy policies and will explain whether SNS marketers conduct ethical practices. This will help create a more sound and efficient way of online social networking conduct that would be beneficial and more secure for users.

To better understand the direct relationship Study I has on a micro-level, additional research would need to be conducted. For example, questions to explore include: how many threats does SNS inoculation withstand until behaviors and attitudes will modify? And to what extent do threats impede behaviors at the time of the rejection? Ivanov et al. (2009) suggest that attacks in inoculation theory may not always act as a booster to increase current behavior. Therefore, results from their study further suggest models be re-examined to explore the extent at which threats may not act as a booster and may influence modified behavior in attitude. 
Future research with a full experimental model including culture or gender difference in explaining the phenomenon will also advance the findings of the current study. Developing an advanced experimental model with different cultural identifiers added to the threatening situation may present different results. Over the past several decades, culture has been used as an important predictor given that culture significantly influences the shaping of one's belief, attitudes, and behavior (Adamopoulos 2004). Developing a comparison model with western and eastern cultures would provide a unique perspective to see how cultures influence our views on the importance of interpersonal relationships on SNS.

Finally, the introduction of additional threats would create a better understanding of how consumers react to different forms of communication presented through SNS (as opposed to communication presented in a person-to-person atmosphere). A modified interpersonal rejection threat which includes privacy as a key element would create an additional influence which may impact the likelihood of an individual to disengage with privacy-threatening activities on SNS usage. For example, Besser and Priel (2010) introduced an achievement failure threat (e.g., career aspirations or the potential to obtain or maintain a job) as one common threat in interpersonal relationships. Introducing an achievement failure threat would expand our knowledge about the effects of threats on the behavioral intention of disengaging from privacythreatening activities on SNS.

In summary, narcissistic tendencies have a great potential to aid in the enhancement of our understanding of SNS privacy concerns and user behaviors. The application of these findings to future research may further develop the level of importance narcissism constructs have on SNS usage behavior. This research developed valuable findings for theory and practice; 
however, a great deal remains to be discovered to understand the full impact of narcissism on individual's SNS behavior. 


\section{REFERENCES}

Abersnagel, Frans (1988), Velten and Musical Mood Induction Procedures: A Comparison with Accessibility of Thought Associations, Behaviour Research and Therapy, 26(1), 79-95.

Adamopoulos, John(2004), "Interpersonal Behavior and Culture," Encyclopedia of Applied Psychology, Retrieved From http://www.credoreference.com.www.libproxy.wvu.edu/entry/estappliedpsyc/interperson al_behavior_and_culture

Ames, Daniel R., Paul Rose, and Cameron P. Anderson (2006), "The NPI-16 As a Short Measure of Narcissism," Journal of Research in Personality, 40, 440-450.

Barnes, Susan B. (2006), 'A Privacy Paradox: Social Networking in the United States', First Monday,11(9), Retrieved from: <http://fi rstmonday.org/htbin/cgiwrap/bin/ojs/ index.php/fm/article/view/1394/1312>

Beckers, John J., and Hank G. Schmidt (2003), “Computer Experience and Computer Anxiety," Computer in Human Behavior, 19(6), 785-797.

Bélanger, France, and Robert E. Crossler (2011), "Privacy in the Digital Age: A Review of Information Privacy Research in Information Systems," MIS Quarterly, 35(4), 117

Bergman, Shawn M., Matthew E. Fearrington, and Shaun W. Davenport, Jacqueline Z. Bergman (2011), "Millenials, Narcissism, and Social Networking: What Narcissists Do On Social Networking Sites and Why," Personality and Individual Differences, 50, 706-711.

Besser, Avi, and Beatriz Priel (2009), "Emotional Responses to a Romantic Partner's Imaginary Rejection: The Roles of Attachment Anxiety, Covert Narcissism, and SelfEvaluation," Journal of Personality, 77(1), 287-325.

Besser, Avi, and Beatriz Priel (2010), "Grandiose Narcissism Versus Vulnerable Narcissism in Threatening Situations: Emotional Reactions to Achievement Failure and Interpersonal Rejection," Journal of Social and Clinical Psychology, 29(8), 874-902.

Bowman, Nicholas D., David Keith Westerman, Chistopher James Claus (2012), "How demanding is social media: Understanding social media diets as a function of perceived costs and benefits - A rational actor perspective," Computers in Human Behavior, 28, $2298-2305$.

Boyd, Danah (2008), "Facebook's Privacy Trainwreck," Convergence: The International Journal of Research into New Media Technologies, 14(1), 13-20.

Carpenter, Christopher J. (2012), "Narcissism on Facebook: Self-Promotional and Anti-Social Behavior," Personality and Individual Differences, 52, 482-486

Chen, Houn-Gee, Charlie Chen, Louis Lox and Samuel Yang (2008), "Online Privacy Control 
Via Anonymity and Pseudonym: Cross-Cultural Implications," Behaviour \& Information Technology, 27(3), $229-242$.

Chen, Kuanchin, and Alan I. Rea (2004), "Protecting Personal Information Online: A Survey of User Privacy Concerns and Control Techniques," The Journal of Computer Information Systems, 44 (4), 85-92.

Corsini, Raymond (1984), "Self-esteem," The Encyclopedia of Psychology, Vol 1, Guilford, CT: DPG Reference, 289-290.

Cohen, Jacob (1983), “The Cost of Dichotomization," Applied Psychological Measurement, 7 , 249-253.

DeWall, C. Nathan, Laura E. Buffardi, Ian Bonser, and W. Keith Campbell (2011), "Narcissism and Implicit Attention Seeking: Evidence From Linguistic Analyses of Social Networking and Online Presentation," Personality and Individual Differences, 51, 57-62.

Downey, Geraldine and Scott Feldman (1996), “Implications of Rejection Sensitivity for Intimate Relationships.” Journal of Personality and Social Psychology, 70(6), 13271343.

Heinssen, Robert K., Carol R. Glass and Luanne A. Knight (1987), “Assessing Computer Anxiety: Development and Validation of the Computer Anxiety Rating Scale," Computers in Human Behavior, 3(1), 49-59.

Hendin, Holly M., and Jonathan M. Cheek (1997), “Assessing Hypersensitive Narcissism: A Reexamination of Murray's Narcissism Scale," Journal of Research in Personality, 31(4), 588-599.

Hof, Robert D. (2011), "You are the Ad,” Technology Review, 114(3), 64-69.

Ivanov, Bobi, Michael Pfau, and Kimberly A. Parker (2009), "Can Inoculation Withstand Multiple Attacks?: An Examination of the Effectiveness of the Inoculation Strategy Compared to the Supportive and Restoration Strategies," Communication Research, 36(5), 655-676.

Kantar Media Company (2011), “Facebook.com”, Retrieved from http://www.compete.com/

Korzaan, Melinda L., and Katherine T. Boswell (2008), "The Influence of Personality Traits and Information Privacy Concerns of Behavioral Intentions," Journal of Computer Information Systems, 48(4), 15-24.

Leary, M. R. (2001). Interpersonal Rejection. Cary, North Carolina: Oxford University Press. Liu, Chang and Kirk P. Amett (2002), "Raising a Red Flag on Global www Privacy Policies," 
Journal of Computer Information Systems, 43(1), 117-127.

Masterson, James F. (1993), The Emerging Self: A Developmental, Self, and Object Relations Approach to the Treatment of the Closet Narcissistic Disorder of the Self. New York: Brunner/Mazel.

Mayo Clinic Staff (2011), Narcissistic Personality Disorder. Retrieved from: http://www.mayoclinic.com/health/narcissistic-personality-disorder/DS00652

McGuire, William (1964), "Inducing resistance to persuasion: Some contemporary approaches," Advances in Experimental Social Psychology, 1, 191-229.

Mehdizadeh, Soraya (2010), "Self-Presentation 2.0: Narcissism and Self-Esteem on Facebook," Cyberpsychology, Behavior, and Social Networking, 13, 357-364.

Miller, Joshua D., Brian J. Hoffman, Eric T. Gaughan, Brittany Gentile, Jessica Maples, and W. Keith Campbell, (2011), "Grandiose and Vulnerable Narcissism: A Nomological Network Analysis," Journal of Personality, 79(5), 1013-1042.

Muftic, Lisa, (2009), "Macro-Micro Theoretical Integration: An Unexplored Theoretical Frontier," Journal of Theoretical and Philosophical Criminology, 1(2), 33-71.

O'Brien, Deirdre and Ann M. Torres (2012), "Social Networking and Online Privacy: Facebook Users' Perceptions," Irish Journal of Management, 31(2), 63-97.

O'Brien, Robert M. (2007), "A Caution Regarding Rules of Thumb for Variance Inflation Factors," Quality and Quantity, 41(5), 673-690.

Parayitam, Satyanarayana, Kiran J. Desai, Mayur S. Desari, and Mary K. Eason (2010), "Computer Attitude as a Moderator in the Relationship Between Computer Anxiety, Satisfaction, and Stress," Computers in Human Behavior, 26(3), 345-352.

Pfau, Michael (2007), "Inoculation Theory," Encyclopedia of Social Psychology, Retrieved from http://www.credoreference.com/entry/sagesocpsyc/inoculation_theory

Ronningstam, Elsa (2009), “Narcissistic Personality Disorder: Facing DSM-V," Psychiatric Annuals, 39(1), 111-121.

Rosenberg, Morris (1965) Society and the adolescent self-image Princeton, New Jersey: Princeton University Press.

Rothery, Grainne (2010), “About Face,” Marketing Age: Business \& Leadership, 4(2), 22-25.

Ryan, Sherry D., Michael Magro, Jason Sharp (2011), "Exploring Educational and Cultural Adaptation through Social Networking Sites," Journal of Information Technology Education: Innovation in Practice, 10, 1-16. 
Shin, Dong-Hee (2010), “The Effects of Trust, Security and Privacy in Social Networking: A Security-Based Approach to Understand the Pattern of Adoption," Interacting with Computers, 22(5), 428-438.

Smith, Jeff H., Sandra J. Milberg, and Sandra J. Burke (1996), "Information Privacy: Measuring Individuals' Concerns About Organizational Practices,” MIS Quarterly, 20(2), 167-197.

Spielberger, Charles, G. Jacobs, S. Russell, and R. Crane (1983), “Assessment of Anger: The State-Trait Anger-Scale," In J. N. Butcher \& C. D. Spielberger (Eds.), Advances in Personality Assessment, 2, 159-187, Hillsdale, NJ: Lawrence Erlbaum Associates, Inc.

Stewart, Kathy A. and Albert H. Segars (2002), “An Empirical Examination of the Concern for Information Privacy Instrument," Information Systems Research, 13(1), 36-49.

Trusov, Michael, Randolph E. Bucklin, Koen Pauwels (2009), "Effects of Word-of-Mouth Versus Traditional Marketing: Findings from an Internet Social Networking Site," Journal of Marketing, 73(5), 90-102.

Utz, Sonja and Nicole Kramer (2009), "The Privacy Paradox on Social Network Sites Revisited: The role of Individual Characteristics and Group Norms," Cyberpsychology: Journal of Psychosocial Research on Cyberspace, 3(2), 1-10.

Wink, Paul (1991), "Two Faces of Narcissism," Journal of Personality and Social Psychology, 61(4), 590-597. 


\section{APPENDIX 1}

\section{Measures}

\section{Grandiose Narcissism:}

I know that I am good because everybody keeps telling me so (OR) When people compliment me I sometimes get embarrassed I like to be the center of attention (OR) I prefer to blend in with the crowd I think I am a special person (OR) I am no better or no worse than most people I like having authority over people (OR) I don't mind following orders I find it easy to manipulate people (OR) I don't like it when I find myself manipulating people I insist upon getting the respect that is due me (OR) I usually get the respect that I deserve I am apt to show off if I get the chance (OR) I try not to be a show off I always know what I am doing (OR) Sometimes I am not sure of what I am doing Everybody likes to hear my stories (OR) Sometimes I tell good stories I expect a great deal from other people (OR) I like to do things for other people I really like to be the center of attention (OR) It makes me uncomfortable to be the center of attention

People always seem to recognize my authority (OR) Being an authority doesn't mean that much to me

I am going to be a great a person (OR) I hope I am going to be successful

I can make anybody believe anything I want them to (OR) People sometimes believe what I tell them

I am more capable than other people (OR) There is a lot that I can learn from other people I am an extraordinary person (OR) I am much like everybody else

\section{Vulnerable Narcissism:}

I can become entirely absorbed in thinking about my personal affairs, my health, my cares or my relations to others.

My feelings are easily hurt by ridicule or by the slighting remarks of others.

When I enter a room I often become self-conscious and feel that the eyes of others are upon me. I dislike sharing the credit of an achievement with others.

I dislike being with a group unless I know that I am appreciated by at least one of those present. I feel that I am temperamentally different from most people.

I often interpret the remarks of others in a personal way.

I easily become wrapped up in my own interests and forget the existence of others.

I feel that I have enough on my hands without worrying about other people's troubles.

I am secretly "put out" when other people come to me with their troubles, asking me for my time and sympathy.

\section{Self-Esteem:}

I feel that I'm a person of worth, at least on an equal plane with others.

I feel that I have a number of good qualities.

All in all, I am inclined to feel that I am a failure. 
I am able to do things as well as most other people.

I feel I do not have much to be proud of.

I take a positive attitude toward myself.

On the whole, I am satisfied with myself.

I wish I could have more respect for myself.

I certainly feel useless at times.

At times I think I am no good at all.

\section{Computer Anxiety:}

I am anxious and concerned about the pace of automation in the world.

Computers are a real threat to privacy in this country.

Sometimes I am afraid the data processing department will lose my data.

I am sometimes frustrated by increasing automation in my home.

\section{Concern for Information Privacy:}

It usually bothers me when companies ask me for personal information.

When companies ask me for personal information, I sometimes think twice before providing it. It bothers me to give personal information to so many companies.

I am concerned that companies are collecting too much personal information about me.

All the personal information in computer databases should be double-checked for accuracy - no matter how much this costs.

Companies should take more steps to make sure that the personal information in their files is accurate.

Companies should have better procedures to correct errors in personal information.

Companies should devote more time and effort to verifying the accuracy of the personal information in their databases.

Companies should not use personal information for any purpose unless it has been authorized by the individuals who provided the information.

When people give personal information to a company for some reason, the company should never use the information for any other purpose.

Companies should never sell the personal information in their computer databases to other companies.

Companies should devote more time and effort to preventing unauthorized access to personal information.

Companies should never share personal information with other companies unless it has been authorized by the individuals who provided the information.

Computer databases that contain personal information should be protected from unauthorized access - no matter how much it costs.

Companies should take more steps to make sure that unauthorized people cannot access personal information in their computers.

\section{Behavioral Intention:}

How likely are you within the NEXT THREE years to... 
Decide not to participate in an online survey, social networking discussion, or join a certain affiliation or group on a social networking site because you do not want to provide certain kinds of information about yourself?

Decide not to open a social networking site account because you do not want to provide certain kinds of information about yourself?

Refuse to give information to social networking sites because you think it is too personal?

Take action to have your name or photos of you removed or placed under more strict privacy settings on social networking sites.

Refuse to use a certain social networking site because you disagree with the way the site uses personal information.

\section{SNS Usage:}

How many hours do you use Social Networking Sites (e.g., Facebook, Twitter) a day?

How many times do you check a Social Networking Site (e.g., Facebook, Twitter) in a given day?

How long would you say your pattern of social network site use has lasted?

Thinking of your social networking usage, would you consider yourself to be a light, medium, or heavy user?

Briefly describe the main reason(s) you use Social Networking Sites.

\section{Manipulation Check:}

To what extent do you feel threatened by the situation above?

To what extent do you feel personally connected to the situation?

To what extent do you get distressed because of the situation above?

\section{State Anger:}

I am mad.

I feel angry.

I am burned up.

I feel irritated.

I feel frustrated.

I am aggravated.

I am annoyed.

\section{Negative State Mood:}

At the moment I feel anxious.

At the moment I feel nervous.

At the moment I feel uneasy.

At the moment I feel tense. 


\section{APPENDIX II \\ Interpersonal Rejection Threat Manipulation Scenarios}

\section{High Threat:}

You get on a Social Networking Site (e.g., Facebook or Twitter) and decide to surprise your friend, $\mathrm{X}$, and write a special message about your friend on your social networking page. As you login and take a look at his/her page, you notice your friend is no longer connected to you (e.g., un-friended you or stopped following you). Later that day you receive a phone call from another friend who tells you the person who is no longer connected to you does not want to associate with you anymore.

\section{Low Threat:}

You get on a Social Networking Site (e.g., Facebook or Twitter) and decide to surprise your friend, $\mathrm{X}$, and write a special message about your friend on your social networking page. As you login and take a look at his/her page, you notice your friend posted a comment that says, "I hate those stupid people" and you immediately think the person is referring to you because of your comments on the Social Networking Site yesterday. Later that day you receive a phone call from your friend, and he/she says that a girl who hurt his/her little sister is very stupid. Now you understand that the 'stupid people' your friend mentioned do not include you. 